\title{
A compact light source providing high-flux, quasi-monochromatic, tunable X-rays in the laboratory
}

Benjamin Hornberger, Jack Kasahara, Martin Gifford, Ronald Ruth, Rod Loewen

Benjamin Hornberger, Jack Kasahara, Martin Gifford, Ronald Ruth, Rod Loewen, "A compact light source providing high-flux, quasi-monochromatic, tunable X-rays in the laboratory," Proc. SPIE 11110, Advances in Laboratorybased X-Ray Sources, Optics, and Applications VII, 1111003 (9 September 2019); doi: 10.1117/12.2527356

Event: SPIE Optical Engineering + Applications, 2019, San Diego, California, United States 


\title{
A compact light source providing high-flux, quasi-monochromatic, tunable X-rays in the laboratory Benjamin Hornberger ${ }^{* a}$, Jack Kasahara ${ }^{\mathrm{a}}$, Martin Gifford ${ }^{\mathrm{a}}$, Ronald Ruth ${ }^{\mathrm{a}}$, Rod Loewen ${ }^{\mathrm{a}}$ aLyncean Technologies, Inc., 47633 Westinghouse Drive, Fremont, CA 94539, USA
}

\begin{abstract}
There is a large performance gap between conventional, electron-impact X-ray sources and synchrotron radiation sources. Electron-impact X-ray sources are compact, low to moderate cost, widely available and can have high total flux, but have limited tunability (broad spectrum bremsstrahlung plus fixed characteristic lines) and low brightness. By contrast, synchrotron radiation sources provide extremely high brightness (coherent flux), are tunable and can be monochromatized to a very high degree. However, they are very large and expensive, and typically operated as national user facilities with limited access. An Inverse Compton Scattering (ICS) X-ray source can bridge this gap by providing a narrow-band, high flux and tunable X-ray source that fits into a laboratory at a cost of a few percent of a large synchrotron facility. It works by colliding a high-power laser beam with a relativistic electron beam, in which case the backscattered photons have an energy in the X-ray regime. This paper will describe the working principle of the Lyncean Compact Light Source, a storage-ring based ICS source, its unique beam properties and recent developments that are expected to increase flux and brightness by an order of magnitude compared to earlier versions. Furthermore, it will illustrate how such an X-ray source can be the cornerstone of a local X-ray facility serving applications from diffraction and imaging to scattering and spectroscopy. An overview of demonstrated and potential applications will be provided.
\end{abstract}

Keywords: X-ray source, Inverse Compton Scattering, Compact Light Source, X-ray diffraction, X-ray imaging, X-ray scattering, X-ray Spectroscopy, table-top synchrotron

\section{INTRODUCTION}

Conventional electron-impact X-ray sources have been in use since the discovery of X-rays by Röntgen in $1895 .{ }^{1}$ They work by accelerating an electron beam onto a metal target (anode), whereby X-rays are generated by two mechanisms resulting in the well-known spectrum of such X-ray sources: Bremsstrahlung ("breaking radiation") generates a continuous spectrum of X-rays with an energy up to the acceleration voltage of the electrons (typically tens to hundreds of keV), while $\mathrm{X}$-ray fluorescence of the atoms in the target generates characteristic lines at specific energies defined by the X-ray emission lines of the target material chosen (typically a few $\mathrm{keV}$ to tens of $\mathrm{keV}$ ).

The X-ray flux or brightness (here loosely defined as flux per source area) is limited by the power, and thus heat load, that the target can tolerate before melting. The most basic type of X-ray source works in reflection geometry, where the X-rays are extracted from the same surface of the (thick) target that the electron beam impinges on. This geometry allows for relatively high power, but the spread of the electrons in the target limits the spot size achievable to the range of 5-10 $\mu \mathrm{m}$ in the case of microfocus reflection sources (high power sources have spot sizes larger than $1 \mathrm{~mm}$ ). Alternatively, in micro/nanofocus transmission sources, the target is a thin metal film on a low-absorbing substrate, and the X-rays are extracted from the opposite side of the target. The limited spread of the electron beam in the thin target allows for spot sizes below $1 \mu \mathrm{m}$ (albeit at very low power), and the geometry allows for very short working distances, useful for applications like micro-CT. To further overcome the limits of heat load on a stationary target, rotating anode sources ${ }^{2}$ have been developed, where the heat load is spread over a larger surface, allowing for much higher power. More recent, advanced developments include liquid metal jet sources ${ }^{3,4}$ and structured anode sources, ${ }^{5}$ but all these developments are reaching the physics limit of what can be achieved with electron-impact sources.

Conventional X-ray sources are ubiquitous and widely commercially available. They are compact, can easily be integrated into cabinet-sized or even desktop instruments, and are affordable (typically from a few tens of thousands of US dollars for simple reflection sources to a few hundred thousand for rotating anode, liquid metal or structured anode sources). They are integrated into many turn-key X-ray instruments serving a wide range of applications, such as:

*benjamin_hornberger@lynceantech.com; phone 16503208300 x427; www.lynceantech.com 
- Medical applications, e.g. standard X-ray, dental X-ray and computed tomography (CT)

- Commercial applications, e.g. baggage inspection

- Industrial applications, e.g. 2D/3D inspection

- Research applications, e.g. X-ray diffraction (XRD), X-ray fluorescence (XRF), Small Angle X-ray Scattering (SAXS), X-ray Microscopy (XRM), or micro-CT

By contrast, synchrotron X-ray sources are based on particle accelerators, where a high-energy (GeV-range) electron beam is circulated in a storage ring. X-rays are generated by transverse acceleration (deflection) of the electron beam in magnetic fields, both in the bending magnets that keep the beam on its quasi-circular trajectory, and in periodic magnet structures (wigglers and undulators), where the X-rays generated by multiple deflections add up to generate even brighter beams (see Figure 1).

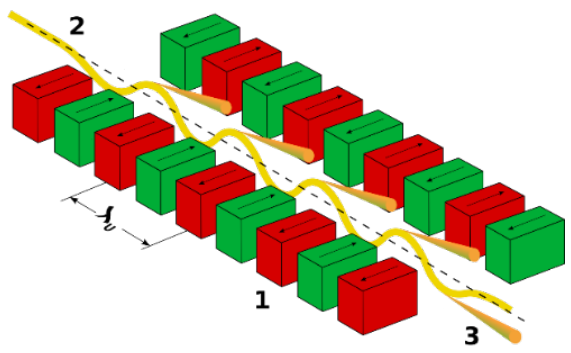

Figure 1. Illustration of an undulator. ${ }^{6}$ An undulator is a periodic structure of dipole magnets (1). An electron beam (2) traversing the structure undergo undulations and produce radiation (3).

Synchrotron sources generate collimated X-ray beams with much higher brightness than is possible with conventional $\mathrm{X}$-ray sources. A more stringent definition of brightness than the one used above is X-ray flux per source area, per solid angle, and per energy band, i.e. it is a measure of beam size achievable, collimated flux and spectral density. While the spectrum from bending magnets and wigglers is broad, the one from undulators is narrower and consists of multiple harmonics. In either case, the high inherent brightness of synchrotrons allows the X-ray beam to be monochromatized to a high degree using crystal or multilayer monochromators. The energy is tunable continuously over a wide range, which can range from well below the X-ray range (IR, UV) to the soft X-ray (hundreds of eV) and hard X-ray $(>100 \mathrm{keV})$ range, depending on the synchrotron design. Synchrotron facilities typically have tens of beamlines providing access to a wide range of X-ray techniques from diffraction and imaging to spectroscopy and scattering.

However, synchrotron facilities require a large investment (hundreds of millions of US dollars) and are therefore typically government funded and operated. Access is provided through competitive proposal systems and limited to a few days of beam time per user per year, although commercial access-for-fee is also available at most facilities. Many beamlines are highly oversubscribed so that only a fraction of the proposals submitted get beamtime assigned. This, along with the limited number of synchrotrons available (about 50 worldwide), presents a barrier of entry that makes synchrotrons inaccessible to many researchers. Furthermore, a single allotment of a few days of beamtime precludes longitudinal experiments, where the same sample needs to be imaged repeatedly over a longer period. Additional restrictions may exist in the ability to measure restricted samples with radiological, biological or other hazards, or where certain types of experimental apparatus are required.

Table 1 provides a qualitative comparison of the X-ray beam properties, general accessibility and applicability to different $\mathrm{X}$-ray techniques between electron-impact and synchrotron sources.

This discussion of conventional and synchrotron X-ray sources indicates that there is a need for X-ray sources that bridge the gap between the two, both in terms of performance (X-ray beam characteristics) and accessibility and price. An X-ray source that provides synchrotron-like beam properties (high flux, monochromaticity, tunability, partial coherence) and that can operate in a local laboratory provides many opportunities by broadening access to advanced X-ray techniques, enabling longitudinal experiments and accommodating restrictive samples. This provides a pathway to industrial and clinical applications that are not feasible at synchrotrons because of the restrictive access model. Furthermore, having a compact source in a local lab provides a synchrotron-like environment that enables training opportunities in the areas of accelerators and instrumentation as well as X-ray techniques and applications. 
In this paper we present the Lyncean Compact Light Source (CLS), an X-ray source based on Inverse Compton Scattering (ICS) that bridges the gap between conventional X-ray sources and synchrotron facilities. The idea of a laser-electron storage ring for the generation of X-rays was conceived by Huang and Ruth in 1997. ${ }^{7}$ Lyncean Technologies, Inc. was founded in 2002 to develop and commercialize the Compact Light Source. X-ray experiments were performed starting in 2007. ${ }^{8-18}$ The first commercial system was delivered to a consortium of the Technical University of Munich (TUM) and the Ludwig-Maximilians-Universität (LMU) and commissioned in 2015. Together with a beamline and two biomedical imaging end-stations developed by TUM researchers, this facility is known as the Munich Compact Light Source (MuCLS). ${ }^{19,20}$ The MuCLS has been operating with a high degree of uptime since then and has enabled many scientific experiments and publications. ${ }^{21-31}$

Table 1. Qualitative comparison of electron-impact and synchrotron X-ray sources. The Lyncean Compact Light Sources bridges the gap for most of these properties.

\begin{tabular}{|c|c|c|}
\hline & Electron-impact X-ray sources & Synchrotron X-ray sources \\
\hline \multicolumn{3}{|l|}{ Beam properties } \\
\hline X-ray flux & Total flux can be high & High \\
\hline Brightness & $\begin{array}{l}\text { Low (moderate for characteristic lines with } \\
\text { rotating anodes, liquid metal jet or other } \\
\text { advanced sources) }\end{array}$ & High \\
\hline $\begin{array}{l}\text { Spectrum and } \\
\text { monochromaticity }\end{array}$ & $\begin{array}{l}\text { Continuum with characteristic lines } \\
\text { Characteristic lines can be monochromatized } \\
\text { (isolated) to some degree }\end{array}$ & $\begin{array}{l}\text { Broad continuum (bending magnet, } \\
\text { wiggler) to narrow peaks (undulator) } \\
\text { Can be monochromatized to high degree }\end{array}$ \\
\hline Tunability & $\begin{array}{l}\text { Limited (choice of target material defines } \\
\text { characteristic line energies; acceleration } \\
\text { voltage sets peak and maximum energy of } \\
\text { continuum) }\end{array}$ & Continuously tunable over wide range \\
\hline Coherence & $\begin{array}{l}\text { Low (moderate coherence for nanofocus or } \\
\text { structured anode sources) }\end{array}$ & High \\
\hline $\begin{array}{l}\text { Beam divergence / } \\
\text { beam size }\end{array}$ & Large & Small \\
\hline \multicolumn{3}{|l|}{ Infrastructure } \\
\hline Size & Compact (cabinet / desktop) & Very large (stadium-size) \\
\hline Cost & Low to moderate & High \\
\hline Accessibility & Commercially available for any lab & $\begin{array}{l}\text { Typically government-funded and operated, } \\
\text { limited access } \\
\text { Only } \sim 50 \text { synchrotrons worldwide }\end{array}$ \\
\hline $\begin{array}{l}X \text {-ray techniques and } \\
\text { application-specific } \\
\text { performance }\end{array}$ & \multicolumn{2}{|c|}{$\begin{array}{l}\text { Generally, most X-ray techniques such as diffraction, imaging, spectroscopy or scattering } \\
\text { can be performed with both electron-impact and synchrotron sources. While the details } \\
\text { depend on the technique and the specific application needs, generally the following } \\
\text { guidelines apply: }\end{array}$} \\
\hline & Longer measurement times & Shorter measurement times \\
\hline & Lower resolution and sensitivity & Higher resolution and sensitivity \\
\hline & $\begin{array}{l}\text { Suitable where fixed energy or } \\
\text { polychromatic beam is acceptable }\end{array}$ & $\begin{array}{l}\text { Required where tunability and/or } \\
\text { monochromaticity is required }\end{array}$ \\
\hline & $\begin{array}{l}\text { Advantageous for large sample sizes (e.g., } \\
\text { imaging of large components and low or } \\
\text { moderate resolution) }\end{array}$ & $\begin{array}{l}\text { Large samples require scanning. Better } \\
\text { suited for high resolution work on smaller } \\
\text { samples }\end{array}$ \\
\hline & $\begin{array}{l}\text { Generally not suited for coherence-based } \\
\text { applications, although phase-contrast } \\
\text { imaging is possible }\end{array}$ & $\begin{array}{l}\text { Well suited for applications with even the } \\
\text { highest coherence requirements (e.g. } \\
\text { nanofocusing or coherent imaging) }\end{array}$ \\
\hline
\end{tabular}




\section{PRINCIPLES OF INVERSE COMPTON SCATTERING}

\subsection{The wave picture - how to shrink an electron storage ring and still generate multi-keV X-rays}

To calculate the X-ray wavelength for a permanent magnet undulator, the undulator equation for the fundamental on-axis can be written as ${ }^{32}$

$$
\lambda_{x, 1}=\frac{\left(1+K^{2} / 2\right)}{2 \gamma^{2}} \lambda_{u}
$$

where $\lambda_{x, 1}$ is the X-ray wavelength of the fundamental, $K$ is the deflection parameter (for undulators, $K \lesssim 1$ ), $\gamma=E_{e} / E_{0}$ is the Lorentz factor (e-beam energy $E_{e}$ in units of electron rest mass energy $E_{0}=0.511 \mathrm{MeV}$ ) and $\lambda_{u}$ is the period of the undulator. For a weak undulator, $K \ll 1$ so that

$$
\lambda_{x, 1}=\frac{\lambda_{u}}{2 \gamma^{2}}
$$

With $\lambda_{u}=1 \mathrm{~cm}$ and $\gamma=6850$ (electron beam energy $E_{e}=3.5 \mathrm{GeV}$ ), the X-ray wavelength becomes $\lambda_{x, 1}=0.11 \mathrm{~nm}$, which corresponds to X-ray energy of $E_{x}=11.6 \mathrm{keV}$. Such storage rings typically have a circumference of several hundred meters. Now, how can we shrink the synchrotron storage ring and still generate multi-keV X-rays? Considering that the magnetic field strength of the bending magnets stays the same, the achievable bending radius is proportional to the e-beam energy. To reduce the circumference of the storage ring by a factor of 100 to a few meters, we need to reduce the e-beam energy, or $\gamma$, by the same amount to about $35 \mathrm{MeV}$. Consequently, we would need to reduce the undulator wavelength by 10,000 to maintain the same X-ray energy. This can be achieved by using a laser beam with a wavelength around $1 \mu \mathrm{m}$ as an undulator - the electromagnetic field of the laser will "undulate" the electron beam in the same way as the periodic arrangement of permanent magnets in a conventional undulator. In fact, the undulator equation for peak photon energy on axis for a laser undulator can be written as ${ }^{33}$

$$
\lambda_{x}=\frac{\lambda_{u}}{4 \gamma^{2}}
$$

The additional factor of 2 in the denominator comes from the fact that the undulator (laser) field is not stationary, but counter-propagates the electron beam at the speed of light. Table 2 below summarizes typical storage ring parameters of synchrotrons and the Lyncean Compact Light Source.

Table 2. Typical storage ring parameters of synchrotrons and the Lyncean Compact Light Source

\begin{tabular}{|l|l|l|l|}
\hline & Synchrotron & Compact Light Source & Scale factor \\
\hline Storage ring diameter & $\sim 60-300 \mathrm{~m}$ & $\sim 1 \mathrm{~m}$ & $\sim 100 \mathrm{x}$ \\
\hline Electron beam energy & $2-8 \mathrm{GeV}$ & $25-50 \mathrm{MeV}$ & $\sim 100 \mathrm{x}$ \\
\hline Bending magnet field strength & $\sim 1 \mathrm{~T}$ & $\sim 1 \mathrm{~T}$ & $\sim$ same \\
\hline Bending magnet emission* & $\mathrm{X}-\mathrm{ray}(\sim \mathrm{keV})$ & Infrared $(\sim 0.1 \mathrm{eV})$ & $\sim 10,000 \mathrm{x}$ \\
\hline Undulator period & $\sim 1 \mathrm{~cm}$ & $\sim 1 \mu \mathrm{m}$ & $\sim 10,000 \mathrm{x}$ \\
\hline Undulator type & Permanent magnet & Laser & \\
\hline
\end{tabular}

* At a traditional synchrotron, typically both bending magnet and undulator / wiggler emission is used for experiments. At the CLS, only the laser undulator radiation is used.

\subsection{The particle picture - inverse Compton scattering}

In the particle picture, this process is known as inverse Compton scattering, where photons in the visible range are backscattered from a beam of relativistic electrons (see Figure $2 \mathrm{a}$ ). The energy of the backscattered photon for arbitrary angles can be calculated as ${ }^{33}$

$$
E_{x}=\frac{E_{L}\left(1-\beta \cos \theta_{i}\right)}{\left(1-\beta \cos \theta_{f}\right)+\left(E_{L} / E_{e}\right)\left[1-\cos \left(\theta_{f}-\theta_{i}\right)\right]}
$$

where $E_{x}$ is the X-ray energy of the backscattered photon, $E_{L}$ is the laser photon energy, $\beta=v / c$ is the ratio of velocity $v$ of the electron to the speed of light $c$ and $\theta_{i}$ and $\theta_{f}$ are incident and final scattering angles, respectively. For a head-on collision $\left(\theta_{i}=180^{\circ}\right)$, small scattering angles $\left(\theta_{f} \ll 1\right), E_{L} \ll E_{0}$ and $\gamma \gg 1$ this simplifies to 


$$
E_{x}=\frac{4 \gamma^{2} E_{L}}{1+\gamma^{2} \theta_{f}^{2}}
$$

The peak X-ray energy for a backscattered photon $\left(\theta_{f}=0^{\circ}\right)$ becomes

$$
E_{x}=4 \gamma^{2} E_{L}
$$

in analogy to Eq (3) above. Figure $2 \mathrm{~b}$ plots peak X-ray energy as a function of electron energy for a laser wavelength of $1064 \mathrm{~nm}$.

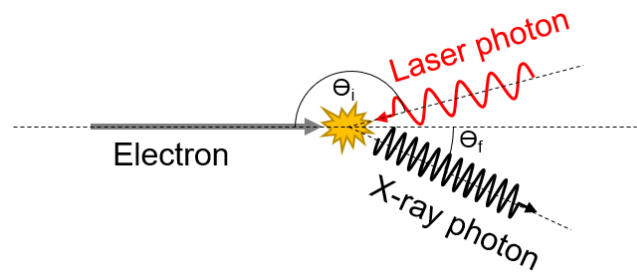

(a)

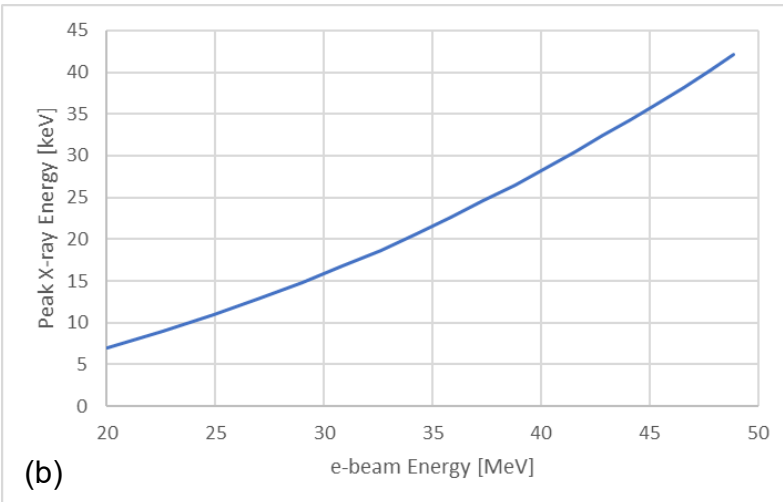

(b)

Figure 2. (a) Schematic of the inverse Compton scattering process. A laser photon collides with a relativistic electron. The backscattered photon has an energy in the X-ray range. (b) Peak X-ray energy (for head-on collision) as a function of electron energy with a laser wavelength of $1064 \mathrm{~nm}$.

If we consider only on-axis electrons $\left(\theta_{i}=180^{\circ}\right)$, we can plot the X-ray energy dependence on scattering angle $\theta_{f}$ from Eq. (5) as shown in Figure 3a. This figure shows how the X-ray energy drops as we move off-axis and indicates how the width of the spectrum depends on the beam divergence that is collected. In practice, the divergence is typically restricted to a few milliradians, which keeps the bandwidth to a few percent.
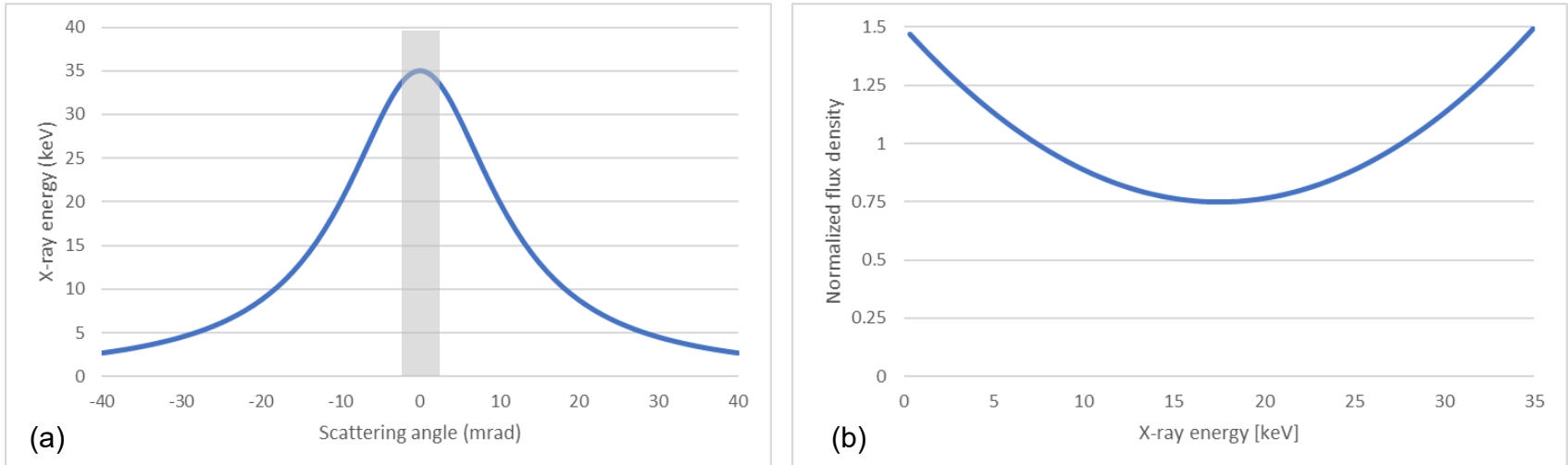

Figure 3. (a) X-ray energy of the scattered photons as a function of scattering angle $\theta_{f}$ for on-axis electrons $\left(\theta_{i}=180^{\circ}\right)$, laser wavelength $1064 \mathrm{~nm}$ and electron energy $44.3 \mathrm{MeV}$ (maximum X-ray energy $35 \mathrm{keV}$ ). In practice, an aperture typically restricts the beam divergence delivered to the experiment to a few milliradians (gray band), so that the resulting beam has a bandwidth of a few percent. (b) Normalized flux density, i.e. probability distribution for the energy (and corresponding scattering angle) of the scattered X-rays.

The case above represents the lowest bandwidth possible in the ideal case of a parallel, monoenergetic electron beam. For an electron beam with finite emittance and energy spread, the spectrum of the X-ray beam is given by the convolution of the scattering angles collected (typically defined by an aperture) and the range of angles of incidence of the electron beam (defined by the e-beam emittance and focusing strength), the energy spread and the flux density 32 (Figure $3 b$ ). Measured spectra for the Lyncean Compact Light Source, which agree well with calculated spectra obtained from Monte-Carlo simulations, are provided in Figure 6 below. 


\subsection{Luminosity}

The photon flux $\dot{N}_{x}$ that can be generated by the inverse Compton process is given by the luminosity formula:

$$
\dot{N}_{x}=\sigma_{\mathrm{Th}} \times \mathcal{L}_{0},
$$

where $\sigma_{\mathrm{Th}}=(8 \pi / 3) r_{e}^{2}$ is the Thomson cross-section and $\mathcal{L}_{0}$ is the luminosity:

$$
\mathcal{L}_{0}=f_{\text {coll }} \frac{N_{L} N_{e}}{2 \pi\left(\sigma_{e}^{2}+\sigma_{l}^{2}\right)}
$$

Here, $f_{\text {coll }}$ is the collision frequency, $N_{L}$ and $N_{e}$ are the number of laser photons and electrons in each pulse, respectively, and $\sigma_{e, l}$ are the transverse spot sizes of the electron and laser beam, respectively, assuming round beams.

The luminosity equation above shows that the X-ray flux in an inverse Compton scattering X-ray source can be optimized by the following:

- $f_{\text {coll }}$ : maximize the photon-electron collision frequency

- $\quad N_{l}$ : maximize laser pulse energy

- $\quad N_{e}$ : maximize electron bunch charge

- $\sigma_{e, l}:$ match and minimize size of e-beam and laser waist

\subsection{LINAC-based vs. storage-ring-based ICS sources}

In general, two approaches exist for ICS sources: linear-accelerator (LINAC) based and storage-ring based.

In the LINAC scheme, low emittance electron bunches from a linear accelerator collide directly with a high intensity pulsed laser, which may be recirculated to enhance flux. The interaction frequency is limited by the repetition frequency of the electron source, which is typically in the tens to hundreds of $\mathrm{Hz}$ range (although schemes exist to increase this to the $\mathrm{kHz}$ range by using pulse trains). To maximize luminosity, the number of electrons per bunch and the laser pulse energy are both increased, and both beams focused to small spots, until practical limits are reached. This approach may be useful for pulse-probe type experiments because they produce low repetition rate X-ray pulses with a large photon count in each pulse and small spot size. LINAC-based approaches are less appropriate for high average intensity applications due to the low repetition rates. Also, since the electron beam is discarded after a single interaction, the power and shielding requirements are substantial, or complex energy-recovery schemes need to be implemented. While several LINAC-based ICS sources have been proposed or are under development, ${ }^{34-39}$ to date none is operational as a user facility for X-ray experiments.

Storage-ring-based sources store the electron bunches on a closed orbit and compensate slow charge decay with infrequent charge injection. Unlike a LINAC source, the electrons are recirculated and can interact over many turns, which keeps power consumption low. The interaction frequency is typically around five orders of magnitude higher ( $65 \mathrm{MHz}$ in the case of the Lyncean Compact Light Source) which makes them quasi-continuous and able to produce much higher average flux and brightness. The cavity-drive laser is matched to the many $\mathrm{MHz}$ rate through the use of a $\mathrm{CW}$ mode-locked laser which is frequency-locked to a high finesse optical cavity (pulse stacking). This cavity technology provides a demonstrated way to circulate up to hundreds of $\mathrm{kW}$ of optical pulse power. Besides the Lyncean Compact Light Source, which is commercially available, several storage-ring-based ICS sources are proposed or under development. ${ }^{40-42}$ A general overview of ICS based sources is provided, for example, in References ${ }^{43,44}$.

\section{THE LYNCEAN COMPACT LIGHT SOURCE}

\subsection{System overview and design}

A simplified schematic of the Lyncean CLS is shown in Figure 4, and a photograph in Figure 5. An electron pulse is generated using an RF photocathode gun. A linear accelerator of about $5 \mathrm{~m}$ in length accelerates the pulse to energies of 25 to $50 \mathrm{MeV}$. The pulse is then injected into a storage ring of about $4.6 \mathrm{~m}$ circumference. Only one electron pulse circulates in the storage ring at a given time. This pulse is dumped and refreshed at a rate of a few tens of $\mathrm{Hz}$. 


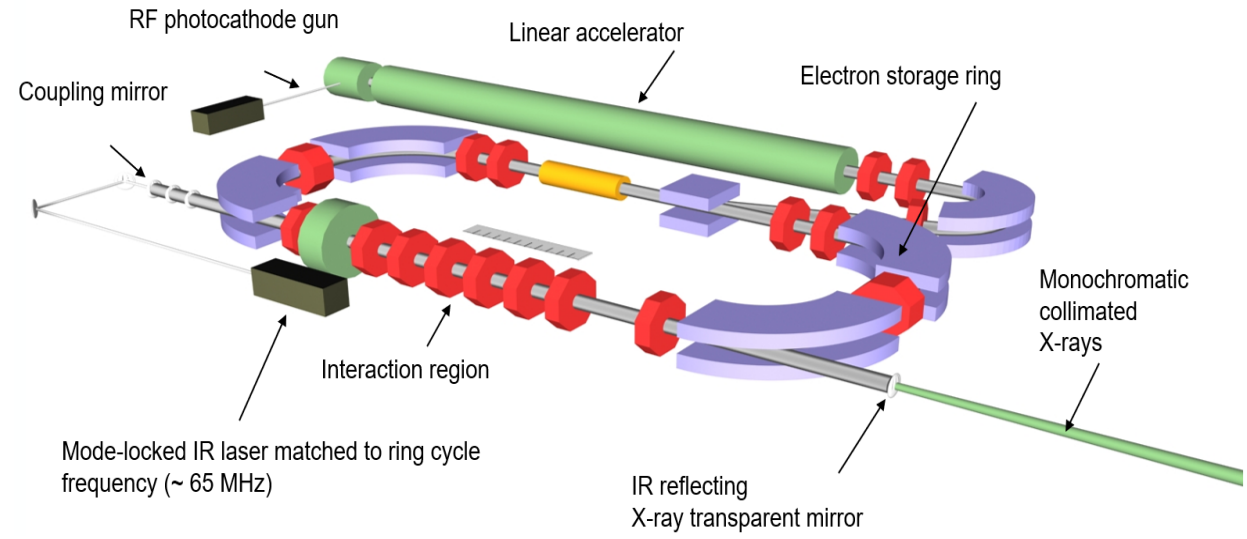

Figure 4. Simplified schematic of the Lyncean Compact Light Source. Electron bunches are generated in the RF photocathode gun, accelerated in the linear accelerator and injected into the electron storage ring. A mode-locked IR laser resonantly drives an optical cavity, shown here schematically as a two-mirror cavity. Electrons and laser pulses collide in the interaction region and generate a collimated, quasi-monochromatic X-ray beam.

The storage ring is configured in a "racetrack" configuration (two 180-degree arcs and two straight sections). One straight section is used for the injection and electron beam dump. In the other straight section (called the Interaction Region), which also serves as one section of a bow-tie optical cavity (see below), the electron beam is focused to a small spot and interacts with an optical pulse to generate X-rays via inverse Compton scattering.

A mode-locked IR laser with wavelength $1 \mu \mathrm{m}$ resonantly drives the optical cavity to build up a high-power laser pulse via pulse-stacking. The enhancement cavity has a gain of about 10,000 , so that a $30 \mathrm{~W}$ average power laser builds up to about $300 \mathrm{~kW}$ of circulating power. The storage ring and the optical cavity share a common leg, where the electron bunch and the laser pulse collide at the interaction point, producing an X-ray pulse directed in a narrow cone in the same direction as the electron beam. The solid angle of the X-ray beam is determined by a limiting aperture at the optical mirror, and the $\mathrm{X}$-rays are coupled out of the vacuum system through a thin membrane.

While the laser wavelength is fixed, the energy of the X-rays produced can be tuned by changing the energy of the electron beam. This is done by changing the magnetic lattice of the storage ring, i.e., by adjusting the magnet currents to provide a closed-orbit solution at a particular energy. The energy of the injected electron beam is tuned to match the ring by adjusting the RF power in the accelerator structures.

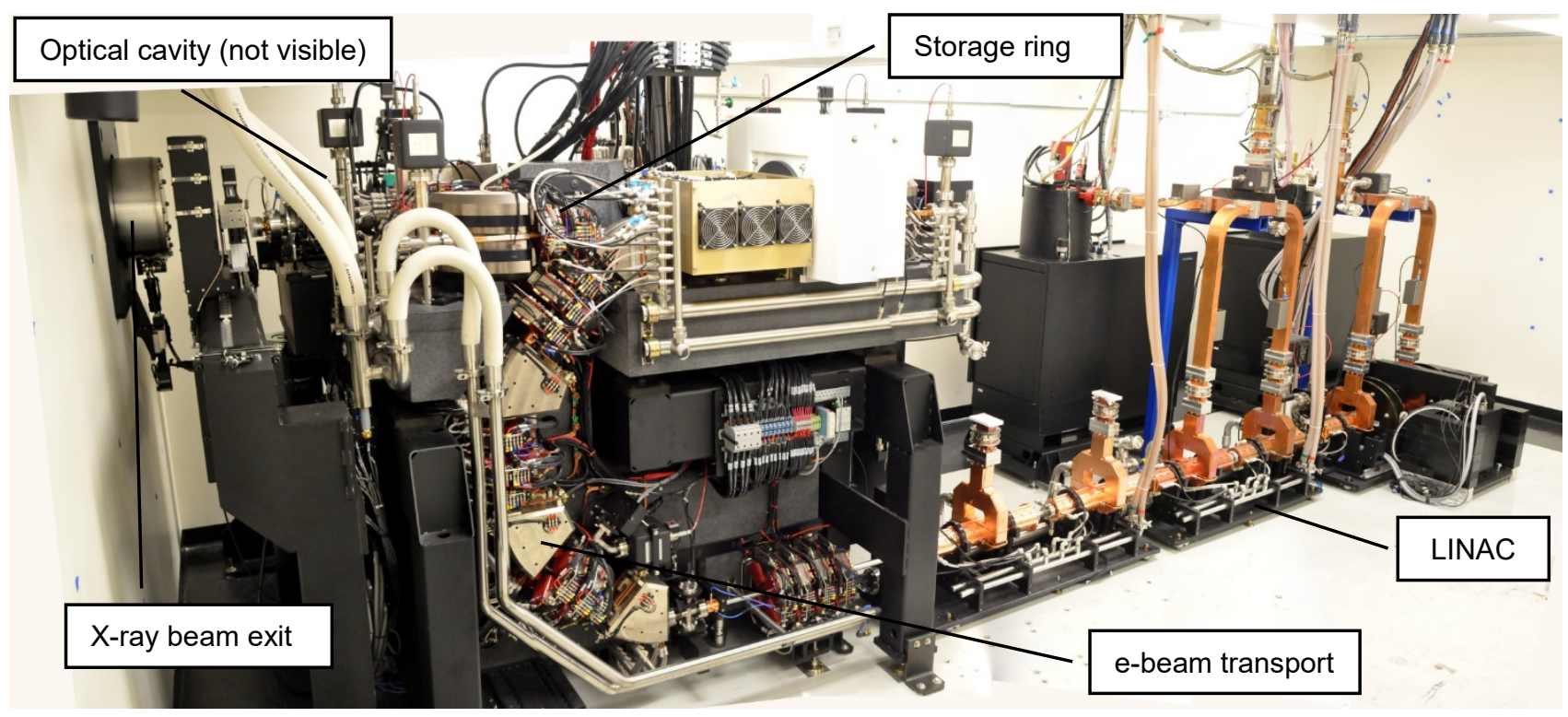

Figure 5. Photograph of the Lyncean Compact Light Source. 


\subsection{Beam properties and parameters}

The X-ray beam parameters of the Lyncean Compact Light Source are as follows and summarized in Table 3.

- A narrow, quasi-monochromatic spectrum with a few percent relative bandwidth (see Figure 6a), suitable for many applications. For applications requiring smaller bandwidth, a monochromator can be implemented.

- Energy tunability in the range of 8-42 keV. Development opportunities exist to shift this energy range to either 4-21 keV, or 16-84 keV (see Section 3.2).

- Partial spatial coherence, advantageous for applications such as propagation- or grating-based phase contrast.

- Small (4-6 mrad) divergence provides a collimated beam:

- Using the direct beam with longer propagation distances enables applications requiring a large beam, such as projection- or grating-based imaging

- Using focusing optics (e.g., Kirkpatrick-Baez type), the beam can be focused down to about 50-100 $\mu \mathrm{m}$ (FWHM) spot size for applications requiring higher flux density, such as diffraction

- Alternatively, collimating optics can provide a quasi-parallel beam for applications such as SAXS.

- Approximately round source, advantageous for applications such as imaging (see Figure 6b).

Table 3. Lyncean Compact Light Source X-ray beam parameters.

\begin{tabular}{|l|l|l|l|}
\hline Parameter & Unit & Current $^{\text {a) }}$ & Under development \\
\hline Total flux (full bandwidth @35 keV) & $\mathrm{ph} / \mathrm{s}$ & $\sim 3 \times 10^{10}$ & $\sim 3 \times 10^{11}$ \\
\hline Source size (FWHM @35 keV) & $\mu \mathrm{m}$ & $\sim 100$ & $\sim 80$ \\
\hline Beam divergence (flattop cone) & $\mathrm{mrad}$ & 4 & 6 \\
\hline Source brightness (full bandwidth @35 keV) & $\mathrm{ph} / \mathrm{s} / \mathrm{mrad}^{2} / \mathrm{mm}^{2}$ & $\sim 4 \times 10^{11}$ & $\sim 4 \times 10^{12}$ \\
\hline X-ray energy range (tunable) & $\mathrm{keV}$ & $15-35$ & $8-42$ \\
\hline X-ray energy bandwidth $(\Delta \mathrm{E} / \mathrm{E}$ FWHM) & $\%$ & $3-5$ & $3-8$ \\
\hline X-ray pulse length (FWHM) & $\mathrm{ps}$ & 140 & 90 \\
\hline X-ray repetition rate & $\mathrm{MHz}$ & 65 & \\
\hline
\end{tabular}

a) Measured beam parameters at Munich Compact Light Source ${ }^{20,45}$

b) Design parameters of next generation CLS currently under development
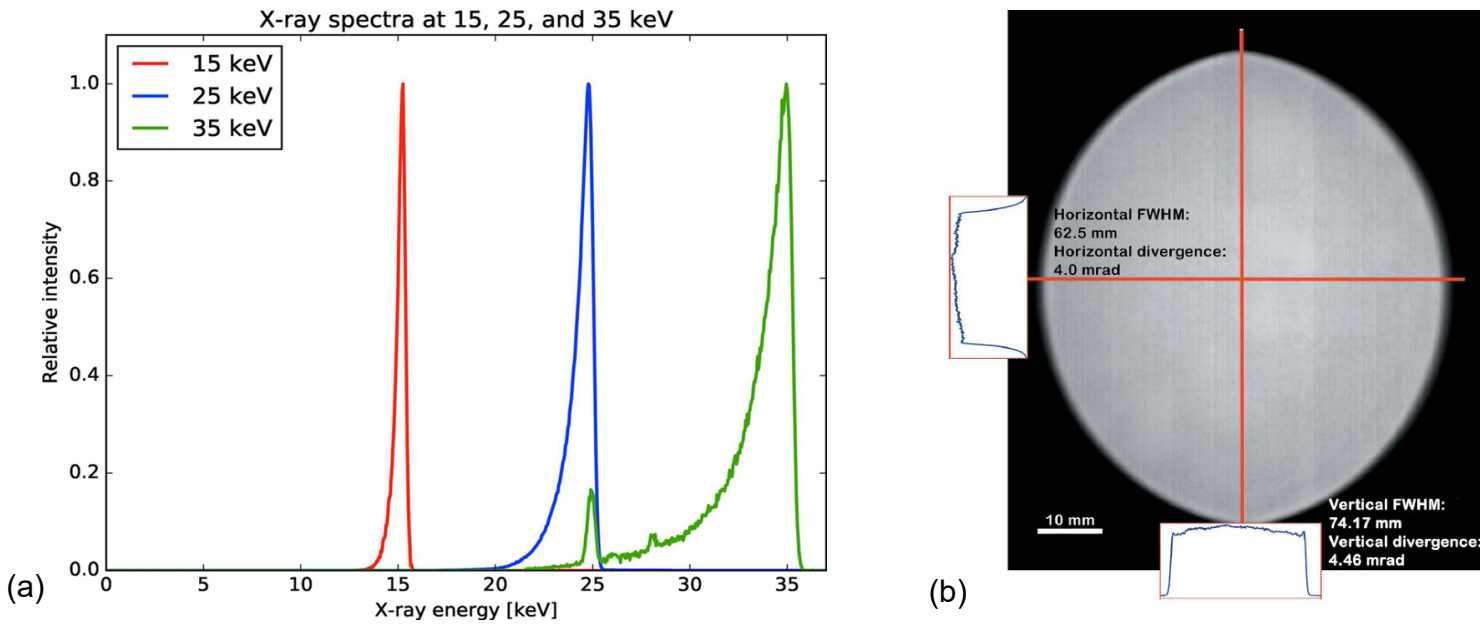

Figure 6. (a) Measured X-ray spectra of the CLS at peak energies $15.2 \mathrm{keV}, 24.8 \mathrm{keV}$ and $35.0 \mathrm{keV}$. The two peaks in the $35 \mathrm{keV}$ spectrum at about 25 and $28 \mathrm{keV}$ are detector artifacts and not part of the CLS X-ray spectrum. (b) Image of the CLS X-ray beam at $16.6 \mathrm{~m}$ distance from the interaction point. Both figures reproduced from Ref. ${ }^{20}$ with permission of the International Union of Crystallography. 


\subsection{Ongoing and future developments}

Several developments are currently underway to increase the performance parameters of the next generation Lyncean Compact Light Source (see Table 3). The storage ring will be upgraded to a multi-bend achromat (MBA) design to lower the emittance and increase the current. At the same time, this will allow increasing the electron beam energy to $50 \mathrm{MeV}$ and thereby the X-ray energy to $42 \mathrm{keV}$. An improved focusing scheme in the optical cavity will reduce the waist of the optical beam. These two improvements combined are expected to increase the brightness by about a factor of 10. Along with an increase in the size of the output aperture of the X-ray beam, which will increase the beam divergence and therefore beam size at the size of the experiment (albeit at broader bandwidth), this will increase the usable X-ray flux by about one order of magnitude as well.

Implementing alternative cavity lasers with $0.5 \mu \mathrm{m}$ or $2 \mu \mathrm{m}$ wavelength allows doubling or halving the X-ray energy, shifting the accessible energy range to $16-84 \mathrm{keV}$ or $4-21 \mathrm{keV}$, respectively. While these are not active developments at the moment, the fundamental technology in terms of suitable drive lasers and optics is available.

Longer term development opportunities include further optimization of the storage ring lattice and increase in optical cavity power, which provide an avenue to further increase brightness and flux by another order of magnitude in the medium term, and to narrow the bandwidth.

\section{CONCEPT FOR A MULTI-DISCIPLINARY X-RAY FACILITY}

A compact synchrotron source can form the cornerstone of a local or regional X-ray facility. With flexible beam conditioning optics and an interchangeable set of endstations, multiple advanced X-ray applications and techniques can be served (see Figure 7). For example, with a set of focusing optics (Figure 8), focused beam experiments such as diffraction or spectroscopy can be performed. The direct, diverging beam close to the source point can be used for high resolution imaging. With a set of collimating optics, a scattering endstation can be placed at an intermediate distance. Finally, at a far distance $(\sim 15 \mathrm{~m})$ and using the direct beam, large field $(>50 \mathrm{~mm})$ imaging, such as grating-based multimodal imaging, can be implemented. With a simple system of kinematic mounts, endstations can be moved in and out of the beam quickly and reproducibly.

Such an X-ray facility brings synchrotron-like beam properties to the local laboratory, spreading out advanced X-ray capabilities regionally, providing increased capacity and broadening access to synchrotron capabilities to local researchers. Local control of experimental time and access allows addressing the need of researchers that have difficulty getting synchrotron beam time. For example, this applies to iterative and longitudinal experiments, experiments requiring many samples for proper statistics, experiments with difficult or restricted samples (e.g., bio- or radiological hazards), or novel techniques still under development. Furthermore, industrial or clinical applications can be developed that are not feasible to become routine under the constraints of synchrotron locations and access models.

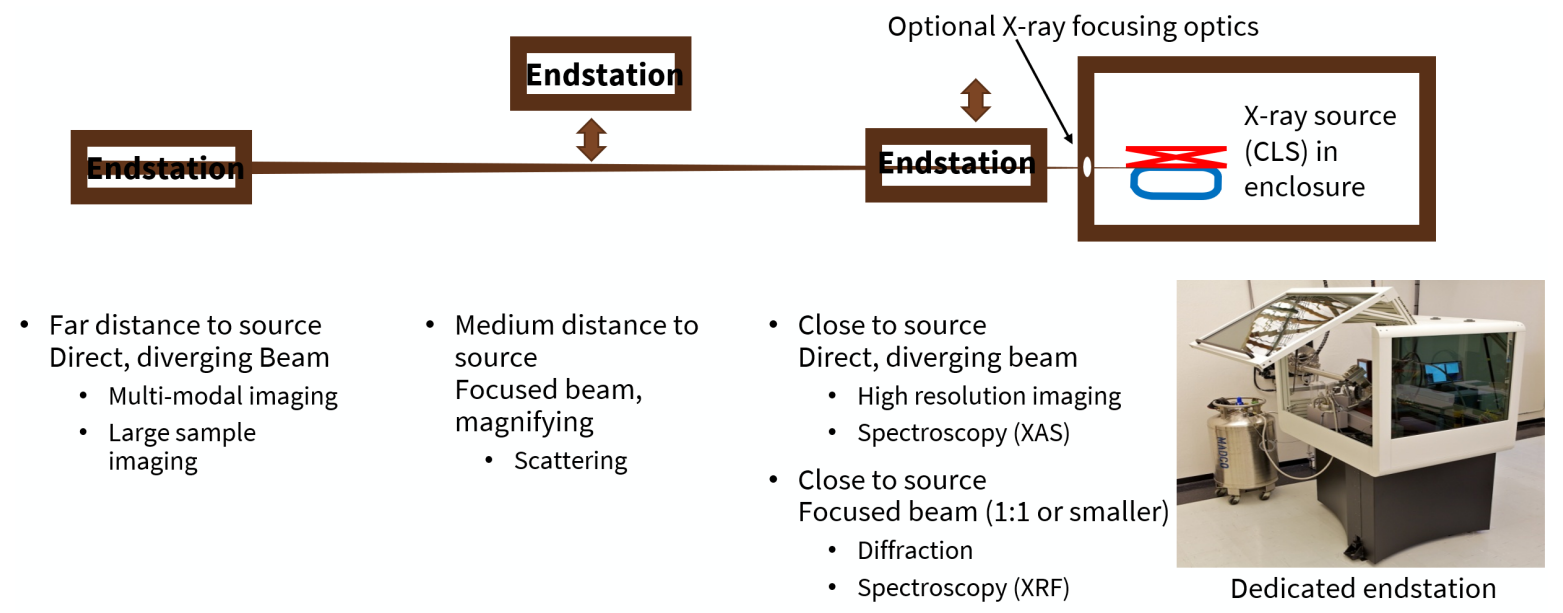

Figure 7. Concept of a multi-disciplinary X-ray facility with a Compact Light Source and a beamline with multiple, interchangeable endstations. When using the direct, diverging beam, the distance from the source point defines the beam size. Alternatively, X-ray focusing optics can be inserted into the beam path (for example located in or near the wall of the radiation enclosure) for focused or collimated beam applications. 

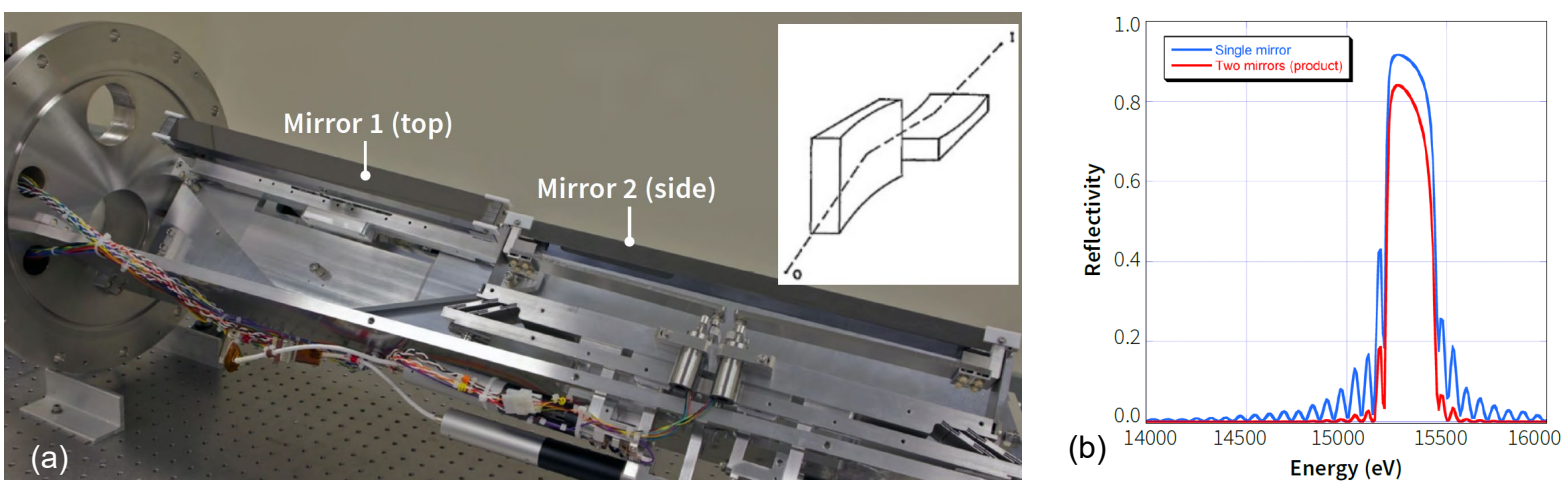

Figure 8. X-ray focusing optics developed for the Lyncean Compact Light Source, consisting of two multilayer-coated mirrors in Kirkpatrick-Baez (KB) focusing configuration. They enable focused beam applications such as diffraction or spectroscopy. (a) Photograph of mirror assembly. Inset: Schematic of KB configuration. (b) Calculated reflectivity of the $\mathrm{KB}$ mirror pair for $15.3 \mathrm{keV}$ X-ray energy. The center of the curve is tunable from 8 to $20 \mathrm{keV}$.

\section{APPLICATION AREAS AND EXAMPLES}

A compact synchrotron such as the Lyncean Compact Light Source can serve all major application areas of X-ray research: Diffraction, imaging, spectroscopy and scattering. In the following, we provide an overview of application examples demonstrated using the Lyncean CLS, and opportunities for new applications.

\subsection{X-ray Diffraction}

Initial demonstration experiments on the Lyncean CLS focused on diffraction applications, specifically macromolecular crystallography ${ }^{9}$ and powder diffraction (unpublished). Opportunities also exist in diffraction for materials science, where the CLS can offer substantially shorter scan times than conventional laboratory systems and enable in situ and some timeresolved experiments. Furthermore, access to higher energies than what is available from the characteristic lines of electron impact sources, along with energy tunability, enables new applications that are not feasible in a lab otherwise.

\subsection{X-ray Imaging}

Given the biomedical imaging research focus of the TUM group, a large number of application examples has been generated in this area. ${ }^{8,10-18,21-26,28-31}$ While earlier experiments focused on demonstrating different X-ray imaging techniques on the CLS, such as mono-energy X-ray absorption tomography, grating-based multimodal imaging, propagation-based phase contrast and phase contrast tomography, these are now applied to specific biomedical applications and extended to advanced techniques, such as dynamic imaging, K-edge subtraction imaging and others.

There is substantial interest in imaging for materials science using the CLS and extending the resolution to the micron, submicron and nanoscale range. Initial paper studies indicate that imaging speeds can be increased by more than a factor of 10 for micro-CT and nano-CT using the CLS, compared to existing laboratory systems, although some technical developments will be necessary to achieve that. These will be explored in the future.

\subsection{X-ray Spectroscopy}

The energy tunability of the CLS lends itself particularly well to providing X-ray absorption spectroscopy (XAS) capabilities in a laboratory setting. Both X-ray Absorption Near-Edge Structure (XANES) and Extended X-ray Absorption Fine Structure (EXAFS) spectroscopy can be performed with the same setup. With a bandwidth of a few percent, energydispersive spectroscopy (ED-XAS) is the most efficient way of implementing this on the CLS. This will also be explored in the future.

\subsection{Small Angle X-ray Scattering (SAXS)}

SAXS for Critical Dimension (CD) measurements on semiconductor samples has been demonstrated in collaboration with NIST (unpublished). 


\section{CONCLUSIONS}

In this paper, we have presented the Lyncean Compact Light Source (CLS), a "mini-synchrotron" X-ray source that bridges the gap between conventional electron-impact sources and synchrotron facilities in terms of X-ray beam characteristics and performance as well as accessibility, cost and infrastructure requirements. It is based on the inverse Compton scattering principle, which in the wave picture can be explained by shrinking the storage ring by a factor of about 100 compared to a typical synchrotron facility, while replacing the permanent magnet undulators with a "laser undulator." In the particle picture, inverse Compton scattering describes the collision of laser photons with relativistic electrons, in which case the backscattered photons have an energy in the X-ray range. The storage-ring approach of the CLS provides a practical, demonstrated way of achieving high average flux and brightness primarily driven by the high repetition rate.

With such an X-ray source, a multi-disciplinary X-ray facility providing many synchrotron-like capabilities can be set up at a local laboratory. One such facility, the Munich Compact Light Source, has been in operation for several years. The CLS can serve a large range of applications across different X-ray techniques such as diffraction, imaging, spectroscopy and scattering. Future developments can further increase X-ray flux and brightness as well as extend the accessible X-ray energy range.

\section{REFERENCES}

[1] Röntgen, W. C., "Ueber eine Neue Art von Strahlen,” Sitzungsberichte der Physikalisch-Medizinischen Gesellschaft zu Würzburg 137, 132-141 (1895).

[2] Skarzynski, T., "Collecting data in the home laboratory: evolution of X-ray sources, detectors and working practices," Acta Crystallographica Section D Biological Crystallography 69(7), 1283-1288 (2013).

[3] Hemberg, O., Otendal, M. and Hertz, H. M., "Liquid-metal-jet anode electron-impact x-ray source," Appl. Phys. Lett. 83(7), 1483-1485 (2003).

[4] Otendal, M., Tuohimaa, T., Vogt, U. and Hertz, H. M., “A 9 keV electron-impact liquid-gallium-jet x-ray source,” Review of Scientific Instruments 79(1), 016102 (2008).

[5] "Sigray Patented x-ray source technologies.", Sigray, <http://www.sigray.com/xcite-microbeam-source-optic> (26 July 2019 ).

[6] "File:Undulator.png - Wikimedia Commons.", <https://commons.wikimedia.org/wiki/File:Undulator.png> (18 October 2018 ).

[7] Huang, Z. and Ruth, R. D., "Laser-Electron Storage Ring," Physical Review Letters 80(5), 976-979 (1998).

[8] Bech, M., Bunk, O., David, C., Ruth, R., Rifkin, J., Loewen, R., Feidenhans'l, R. and Pfeiffer, F., "Hard X-ray phase-contrast imaging with the Compact Light Source based on inverse Compton X-rays," Journal of Synchrotron Radiation 16(1), 43-47 (2009).

[9] Abendroth, J., McCormick, M. S., Edwards, T. E., Staker, B., Loewen, R., Gifford, M., Rifkin, J., Mayer, C., Guo, W., Zhang, Y., Myler, P., Kelley, A., Analau, E., Hewitt, S. N., Napuli, A. J., Kuhn, P., Ruth, R. D. and Stewart, L. J., "X-ray structure determination of the glycine cleavage system protein $\mathrm{H}$ of Mycobacterium tuberculosis using an inverse Compton synchrotron X-ray source," Journal of Structural and Functional Genomics 11(1), 91100 (2010).

[10] Bech, M., Schleede, S., Potdevin, G., Achterhold, K., Bunk, O., Jensen, T. H., Loewen, R., Ruth, R. and Pfeiffer, F., "Experimental validation of image contrast correlation between ultra-small-angle X-ray scattering and gratingbased dark-field imaging using a laser-driven compact X-ray source," Photonics \& Lasers in Medicine 1(1), 47-50 (2012).

[11] Schleede, S., Meinel, F. G., Bech, M., Herzen, J., Achterhold, K., Potdevin, G., Malecki, A., Adam-Neumair, S., Thieme, S. F., Bamberg, F., Nikolaou, K., Bohla, A., Yildirim, A. O., Loewen, R., Gifford, M., Ruth, R., Eickelberg, O., Reiser, M. and Pfeiffer, F., "Emphysema diagnosis using X-ray dark-field imaging at a laserdriven compact synchrotron light source," Proceedings of the National Academy of Sciences 109(44), 1788017885 (2012).

[12] Schleede, S., Bech, M., Achterhold, K., Potdevin, G., Gifford, M., Loewen, R., Limborg, C., Ruth, R. and Pfeiffer, F., "Multimodal hard X-ray imaging of a mammography phantom at a compact synchrotron light source," Journal of Synchrotron Radiation 19(4), 525-529 (2012).

[13] Achterhold, K., Bech, M., Schleede, S., Potdevin, G., Ruth, R., Loewen, R. and Pfeiffer, F., "Monochromatic computed tomography with a compact laser-driven X-ray source,” Scientific Reports 3, 1313 (2013). 
[14] Meinel, F. G., Schwab, F., Schleede, S., Bech, M., Herzen, J., Achterhold, K., Auweter, S., Bamberg, F., Yildirim, A. Ö., Bohla, A., Eickelberg, O., Loewen, R., Gifford, M., Ruth, R., Reiser, M. F., Pfeiffer, F. and Nikolaou, K., "Diagnosing and Mapping Pulmonary Emphysema on X-Ray Projection Images: Incremental Value of GratingBased X-Ray Dark-Field Imaging,” PLoS ONE 8(3), C. Taube, Ed., e59526 (2013).

[15] Schwab, F., Schleede, S., Hahn, D., Bech, M., Herzen, J., Auweter, S., Bamberg, F., Achterhold, K., Yildirim, A. Ö., Bohla, A., Eickelberg, O., Loewen, R., Gifford, M., Ruth, R., Reiser, M. F., Nikolaou, K., Pfeiffer, F. and Meinel, F. G., "Comparison of Contrast-to-Noise Ratios of Transmission and Dark-Field Signal in Grating-Based X-ray Imaging for Healthy Murine Lung Tissue,” Zeitschrift für Medizinische Physik 23(3), 236-242 (2013).

[16] Fu, J., Schleede, S., Tan, R., Chen, L., Bech, M., Achterhold, K., Gifford, M., Loewen, R., Ruth, R. and Pfeiffer, F., "An algebraic iterative reconstruction technique for differential X-ray phase-contrast computed tomography," Zeitschrift für Medizinische Physik 23(3), 186-193 (2013).

[17] Eggl, E., Schleede, S., Bech, M., Achterhold, K., Loewen, R., Ruth, R. D. and Pfeiffer, F., "X-ray phase-contrast tomography with a compact laser-driven synchrotron source," Proceedings of the National Academy of Sciences 112(18), 5567-5572 (2015).

[18] Eggl, E., Schleede, S., Bech, M., Achterhold, K., Grandl, S., Sztrókay, A., Hellerhoff, K., Mayr, D., Loewen, R., Ruth, R. D., Reiser, M. F. and Pfeiffer, F., "X-ray phase-contrast tomosynthesis of a human ex vivo breast slice with an inverse Compton x-ray source," EPL (Europhysics Letters) 116(6), 68003 (2016).

[19] “Munich Compact Light Source (MuCLS).”, MSB: Munich Compact Light Source, $<$ https://www.bioengineering.tum.de/infrastructure/munich-compact-light-source/> (27 September 2018 ).

[20] Eggl, E., Dierolf, M., Achterhold, K., Jud, C., Günther, B., Braig, E., Gleich, B. and Pfeiffer, F., "The Munich Compact Light Source: initial performance measures," Journal of Synchrotron Radiation 23(5), 1137-1142 (2016).

[21] Eggl, E., Mechlem, K., Braig, E., Kulpe, S., Dierolf, M., Günther, B., Achterhold, K., Herzen, J., Gleich, B., Rummeny, E., Noël, P. B., Pfeiffer, F. and Muenzel, D., "Mono-Energy Coronary Angiography with a Compact Synchrotron Source," Scientific Reports 7, 42211 (2017).

[22] Gradl, R., Dierolf, M., Hehn, L., Günther, B., Yildirim, A. Ö., Gleich, B., Achterhold, K., Pfeiffer, F. and Morgan, K. S., "Propagation-based Phase-Contrast X-ray Imaging at a Compact Light Source," Scientific Reports 7, 4908 (2017).

[23] Töpperwien, M., Gradl, R., Keppeler, D., Vassholz, M., Meyer, A., Hessler, R., Achterhold, K., Gleich, B., Dierolf, M., Pfeiffer, F., Moser, T. and Salditt, T., "Propagation-based phase-contrast x-ray tomography of cochlea using a compact synchrotron source," Scientific Reports 8, 4922 (2018).

[24] Jud, C., Braig, E., Dierolf, M., Eggl, E., Günther, B., Achterhold, K., Gleich, B., Rummeny, E., Noël, P., Pfeiffer, F. and Muenzel, D., "Trabecular bone anisotropy imaging with a compact laser-undulator synchrotron x-ray source," Scientific Reports 7, 14477 (2017).

[25] Gradl, R., Dierolf, M., Günther, B., Hehn, L., Möller, W., Kutschke, D., Yang, L., Donnelley, M., Murrie, R., Erl, A., Stoeger, T., Gleich, B., Achterhold, K., Schmid, O., Pfeiffer, F. and Morgan, K. S., "In vivo Dynamic PhaseContrast X-ray Imaging using a Compact Light Source," Scientific Reports 8, 6788 (2018).

[26] Gradl, R., Morgan, K. S., Dierolf, M., Jud, C., Hehn, L., Gunther, B., Moller, W., Kutschke, D., Yang, L., Stoeger, T., Pfeiffer, D., Gleich, B., Achterhold, K., Schmid, O. and Pfeiffer, F., "Dynamic in vivo chest X-ray dark-field imaging in mice," IEEE Transactions on Medical Imaging 38(2), 649-656 (2019).

[27] Burger, K., Ilicic, K., Dierolf, M., Günther, B., Walsh, D. W. M., Schmid, E., Eggl, E., Achterhold, K., Gleich, B., Combs, S. E., Molls, M., Schmid, T. E., Pfeiffer, F. and Wilkens, J. J., "Increased cell survival and cytogenetic integrity by spatial dose redistribution at a compact synchrotron X-ray source," PLOS ONE 12(10), G. E. Woloschak, Ed., e0186005 (2017).

[28] Eggl, E., Grandl, S., Sztrókay-Gaul, A., Dierolf, M., Jud, C., Heck, L., Burger, K., Günther, B., Achterhold, K., Mayr, D., Wilkens, J. J., Auweter, S. D., Gleich, B., Hellerhoff, K., Reiser, M. F., Pfeiffer, F. and Herzen, J., "Dose-compatible grating-based phase-contrast mammography on mastectomy specimens using a compact synchrotron source," Scientific Reports 8, 15700 (2018).

[29] Braig, E., Böhm, J., Dierolf, M., Jud, C., Günther, B., Mechlem, K., Allner, S., Sellerer, T., Achterhold, K., Gleich, B., Noël, P., Pfeiffer, D., Rummeny, E., Herzen, J. and Pfeiffer, F., "Direct quantitative material decomposition employing grating-based X-ray phase-contrast CT," Scientific Reports 8, 16394 (2018).

[30] Kulpe, S., Dierolf, M., Braig, E., Günther, B., Achterhold, K., Gleich, B., Herzen, J., Rummeny, E., Pfeiffer, F. and Pfeiffer, D., "K-edge subtraction imaging for coronary angiography with a compact synchrotron X-ray source," PLOS ONE 13(12), e0208446 (2018). 
[31] Gradl, R., Dierolf, M., Yang, L., Hehn, L., Günther, B., Möller, W., Kutschke, D., Stoeger, T., Gleich, B., Achterhold, K., Donnelley, M., Pfeiffer, F., Schmid, O. and Morgan, K. S., "Visualizing treatment delivery and deposition in mouse lungs using in vivo x-ray imaging," Journal of Controlled Release 307, 282-291 (2019).

[32] Hofmann, A., [The Physics of Synchrotron Radiation, digital printed version], Cambridge Univ. Press, Cambridge (2007).

[33] Loewen, R., "A Compact Light Source: Design and Technical Feasibility Study of a Laser-Electron Storage Ring X-Ray Source,” SLAC-R-632, 826755 (2004).

[34] Urakawa, J., "Development of a compact X-ray source based on Compton scattering using a $1.3 \mathrm{GHz}$ superconducting RF accelerating linac and a new laser storage cavity," Nuclear Instruments and Methods in Physics Research Section A: Accelerators, Spectrometers, Detectors and Associated Equipment 637(1), S47-S50 (2011).

[35] Hajima, R., Kikuzawa, N., Nishimori, N., Hayakawa, T., Shizuma, T., Kawase, K., Kando, M., Minehara, E., Toyokawa, H. and Ohgaki, H., "Detection of radioactive isotopes by using laser Compton scattered -ray beams," Nuclear Instruments and Methods in Physics Research Section A: Accelerators, Spectrometers, Detectors and Associated Equipment 608(1), S57-S61 (2009).

[36] Graves, W. S., Brown, W., Kaertner, F. X. and Moncton, D. E., "MIT inverse Compton source concept," Nuclear Instruments and Methods in Physics Research Section A: Accelerators, Spectrometers, Detectors and Associated Equipment 608(1), S103-S105 (2009).

[37] Graves, W. S., Bessuille, J., Brown, P., Carbajo, S., Dolgashev, V., Hong, K.-H., Ihloff, E., Khaykovich, B., Lin, H., Murari, K., Nanni, E. A., Resta, G., Tantawi, S., Zapata, L. E., Kärtner, F. X. and Moncton, D. E., "Compact x-ray source based on burst-mode inverse Compton scattering at $100 \mathrm{kHz}$," Phys. Rev. ST Accel. Beams 17(12), 120701 (2014).

[38] Graves, W. S. and others., “ASU Compact XFEL," Proc. of International Free Electron Laser Conference (FEL'17), Santa Fe, NM, USA, August 20-25, 2017, 225-228, JACoW, Geneva, Switzerland (2018).

[39] Stragier, X. F. D., Mutsaers, P. H. A. and Luiten, O. J., "Smart*Light: A Tabletop, High Brilliance, Monochromatic and Tunable Hard X-ray Source for Imaging and Analysis.," Microscopy and Microanalysis 24(S2), 314-315 (2018).

[40] Variola, A., "The ThomX Project," 2nd International Particle Accelerator Conference (IPAC'11) WEOAA01, 1903-1905, Joint Accelerator Conferences Website, San Sebastian, Spain (2011).

[41] Xu, H. S., Huang, W. H., Tang, C. X. and Lee, S. Y., "Design of a 4.8-m ring for inverse Compton scattering xray source," Phys. Rev. ST Accel. Beams 17(7), 070101 (2014).

[42] V. Androsov, N. Ayzatsky, V. Boriskin, E. Bulyak, A. Dovbnya, I. Drebot, P. Gladkikh, A. Gordienko, V. Grevtsev, A. Gvozd, V. Ivashenko, A. Kalamayko, I. Karnaukhov, I. Karnaukhov, V. Kozin, V. Kushnir, V. Lyashchenko, V. Margin, N. Mocheshnikov, et al., "The Kharkov X-Ray Generator Facility NESTOR," Proceedings of IPAC2013, 2253-2255, Shanghai, China (2013).

[43] Jacquet, M., "Potential of compact Compton sources in the medical field," Physica Medica 32(12), 1790-1794 (2016).

[44] Hajima, R., "Status and Perspectives of Compton Sources," Physics Procedia 84, 35-39 (2016).

[45] Eggl, E., "Biomedical X-Ray Imaging at the Munich Compact Light Source," PhD Thesis (2017). 